\title{
1 Active neural coordination of motor behaviors with internal states
}

2

3

4

5

6

8

3

Yisi S. Zhang ${ }^{1}$, Daniel Y . Takahashi1,2, Ahmed El Hady ${ }^{1,3,4}$, Diana A. Liao ${ }^{1}$, Asif A. Ghazanfar ${ }^{1,5}$

${ }^{1}$ Princeton Neuroscience Institute, Princeton University, Princeton NJ 08544, USA

${ }^{2}$ Brain Institute, Federal University of Rio Grande do Norte, Natal RN 59076-550, Brazil

${ }^{3}$ Center for Advanced Study of Collective Behavior, University of Konstanz, Konstanz 78464, Germany

${ }^{4}$ Department of Collective Behavior, Max Planck Institute of Animal Behavior, Konstanz 78464, Germany

${ }^{5}$ Department of Psychology, Princeton University, Princeton, NJ 08544, USA 


\begin{abstract}
1 Abstract
2

3 The brain continuously coordinates skeletomuscular movements with internal physiological states like arousal, 4 but how is this coordination achieved? One possibility is that brain simply reacts to changes in external 5 and/or internal signals. Another possibility is that it is actively coordinating both external and internal 6 activities. We used functional ultrasound imaging to capture a large medial section of the brain, including 7 multiple cortical and subcortical areas, in marmoset monkeys while monitoring their spontaneous movements 8 and cardiac activity. By analyzing the causal ordering of these different time-series, we found that information 9 flowing from the brain to movements and heart rate fluctuations were significantly greater than in the 10 opposite direction. The brain areas involved in this external versus internal coordination were spatially 11 distinct but also extensively interconnected. Temporally, the brain alternated between network states for this 12 regulation. These findings suggest that the brain's dynamics actively and efficiently coordinate motor behavior 13 with internal physiology.
\end{abstract}

14 
Animals and humans continuously regulate physiology and behavior to maintain stability, i.e., keep physiological variables within a tenable range (1). This regulation not only involves triggering autonomic reflexes that directly adjust physiological processes such as heart rate, glucose level, and body temperature but also directs skeletomotor behaviors to interact with the external world that affect physiological states (e.g., feeding, locomotion, social interaction)(2,3). The changing internal states of the body remodel sensorimotor interactions with the external environment on various timescales (4). For example, the phase of the cardiac cycle influences the emotional processing of faces (5), vocal interactions are correlated with autonomic oscillations (6-8), and hunger can modulate the switch between sleep versus foraging behaviors (9). In all cases, the internal physiological states must be coordinated with motor behaviors through the dynamics of large-scale networks of cortical and subcortical regions, but how?

Based on the spectrum of cytoarchitectonic differentiation, one proposal is that the mammalian brain follows a 'centrifugal' cortical organization from the outer side (the primary sensory and motor areas) to the inside. Inside areas include the heteromodal, paralimbic and limbic regions that, in humans, overlap substantially with the default mode network (10-12). The outside areas directly regulate the interactions with the external environment, while the inside areas are associated with autonomic functions regulating the internal milieu through subcortical areas, primarily the hypothalamus (12-14). In humans, functional imaging and gene expression analyses reveal a division of the brain into two cortical networks corresponding to this external-internal dichotomy $(15,10)$. Such a brain architecture entails an embodied account of perception, emotion, and decision making (17-22).

However, within such a framework, how are motor behaviors and the internal state of the body organized with the brain's activity in space and time? If we assume a context in which there are no overt external sensory signals, and the animal coordinates interactions with external and internal environments by its intrinsic dynamics, then there are currently three hypotheses for the inter-relationships between brain activity, motor behavior, and internal physiology (Fig. 1A). The first hypothesis postulates that spontaneous motor behaviors--like twitches, facial movements, and fidgets--drive the large-scale activity of the brain (2326). The behavior-related wide-spread brain activity could originate from re-afferent sensory input and may facilitate contextualized signal processing (27). Under this hypothesis, there are two possibilities for the internal physiological state: it is either a follower of movements through peripheral regulation (Hypothesis \#1.1) or centrally regulated (Hypothesis \#1.2). In the second hypothesis, brain dynamics are passive responses to ongoing physiological states and provide signals--including those to produce behaviors--to maintain homeostasis of the body (Hypothesis \#2) (28-30). The third hypothesis proposes that the brain not only actively predicts signals of the external world $(31,32)$ but also the interoceptive signals of the body for homeostatic control (Hypothesis \#3) (33-35).

To test these hypotheses, we studied the inter-relationships between the brain, external (spontaneous movement), and internal (cardiac) activities in marmoset monkeys (Callithrix jacchus) under a task-absent context. These various activities are prevalent on the timescale of tens of seconds $(23,36-40)$, and thus can be tested in experimental sessions lasting for 10-20 minutes. We simultaneously used functional ultrasound (fUS) imaging of the midsagittal plane of the marmoset brain, along with videos of behaviors and measures of heart rate (electrocardiogram, ECG) to establish: 1) the direction of information flow between the three components, and 2) the spatial and the temporal organizations of brain activity as a function of spontaneous movements and cardiac changes.

\section{Results}

Subjects $(\mathrm{n}=3)$ were placed in a partial restraint device to allow for both stable neural imaging and movements of most of the body. Using behavioral videography analysis (35 sessions, 27259 seconds of recording), we detected occasional movements of the limbs, tail, and body, as well as facial movements, including blinks and movements of ear and mouth (Fig. 1B). Movements were also quantified by the motion intensity (MI) regardless of the type. In parallel, we carried out electrocardiography (ECG) for continuous 
heart rate recording (Fig. 1C). The occurrence of different behaviors varied coherently with heart rate to different degrees with all, except ear movements, showing a peak around $0.03 \mathrm{~Hz}$ (test against the 95\% confidence interval (CI) of phase-randomized surrogates; Fig. 1D). Using MI alone, the coherence between movement and heart rate could also be well captured (Fig. 1D), and there was no temporal difference between MI and elaborated movements with respect to heart rate (Fig. S1). Thus, for ease of presentation, we used MI to represent all kinds of movements in the following analyses.

A

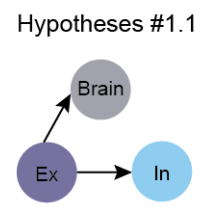

Hypotheses \#2

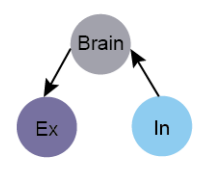

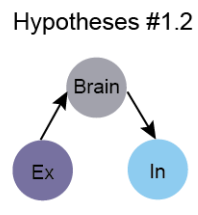

Hypotheses \#3

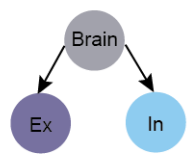

B

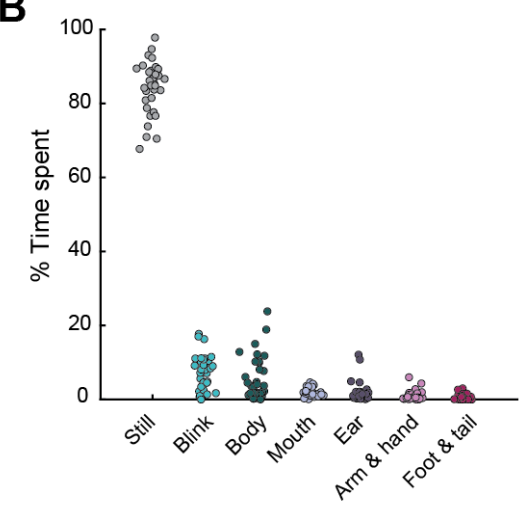

D

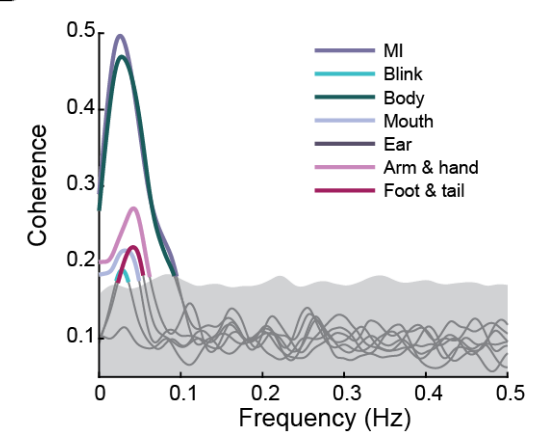

C

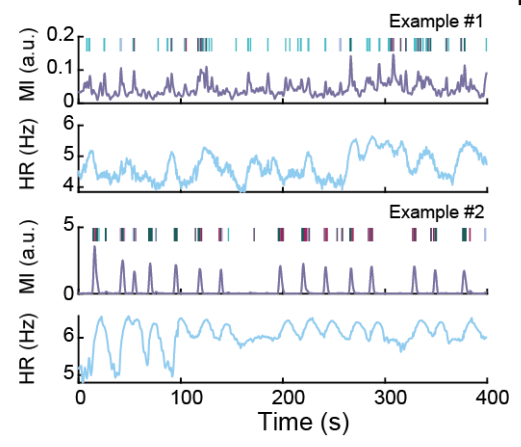

Figure 1. Organization of external and internal activities. (A) Candidate hypotheses on the interrelationships between brain activity, movement and heart rate fluctuation. (B) Percentage time spent on different types of movements. Each point is a session. Jitters are added to help visualize. (C) Two exemplar data of movement type, MI, and HR. Types of movements are color-coded similarly to (B). Note that small MI variations correspond to facial movements such as blinks and large MIs correspond to movements of the body parts. (D) Coherence between each HR-movement type pair and the HR-MI pair. Highlighted segments are significantly higher than the $95 \% \mathrm{Cl}$ of the phaserandomized surrogates.

Meanwhile, we measured brain activity in a large portion of the midsagittal plane, including cortical and subcortical areas using fUS (35 sessions; Fig. 2A). fUS measures cerebral blood volume (CBV) dynamics in the microvasculature, an indirect measure of neuronal activity(41-43), with a large field of view (FOV) (16 $\mathrm{mm}(\mathrm{AP}) \times 20 \mathrm{~mm}(\mathrm{DV}))$, a spatial resolution of $130 \mu \mathrm{m}(\mathrm{AP}) \times 125 \mu \mathrm{m}(\mathrm{DV})$, and a frame rate of $2 \mathrm{~Hz}$. The FOV covered primary sensory and motor areas: the medial motor (M1) and somatosensory (SS); motor associate areas: pre-supplementary motor area (preSMA) and supplemental motor area (SMA); highheteromodal and paralimbic areas: prefrontal (PFC), mid-cingulate cortices (MCC); as well as cortices constituting the DMN: the ventromedial prefrontal cortex (vmPFC) and posterior cingulate cortex (PCC) (44). It also covered subcortical areas relevant to autonomic functions(45), including the dorsal and ventral lateral septal nuclei (LSD and LSV), preoptic area (POA), ventromedial hypothalamus (VMH), posterior hypothalamus (PH), mediodorsal nucleus of the thalamus (MD), habenula nuclei (Hb) and part of the reticular formation (RF) (Fig. 2A). Thus, the simultaneous activities of many brain areas (though not all of them) highly relevant to motor behavior and internal physiology were incorporated in the measurement.

To evaluate the relationship between brain and peripheral activities, we first parcellated the image into regions of interest (ROIs) based on fUS signals. We estimated a group-level parcellation using all 
1 available fUS data based on group independent component analysis (4 subjects, 60 sessions, 85427 frames; Methods), and segregated the brain section into 19 ROIs (Fig. 2B, C). Among the 19 ROIs, 14 were identified as non-vessel ROIs and were named based on corresponding anatomical annotations after aligning to a reference brain atlas (Methods; Fig. 2C, Fig. S2) (46, 47). The brain parcellation results were consistent across subjects (Fig. 2C), and thus we concatenated the ROI signals across all sessions.

The information flow between time-series of brain activity, movements (MI), and heart rate was assessed using block partial directed coherence (bPDC), a measure of information flow in the frequency domain (48) and invariant to causal filtering like the hemodynamic response function (see Supplementary text) (49). The bPDC treated the ROI signals as one multivariate time-series network node and the movement and heart rate as the other two nodes (Fig. 2D). We found that the bPDC was significant in both directions between brain and movement, and between brain and heart rate; while the bPDC between movement and heart rate was not significant, suggesting that their coordination was via the brain (test against the $95 \%$ confidence intervals (CI) of phase-randomized surrogates, Bonferroni corrected; Fig. 2E). Hence, we could reject Hypothesis \#1.1 as the information flow between movement and heart rate was indirect. We then tested if the brain activity was driven more by the periphery or vice versa by comparing the total information flow in each direction, which was calculated by integrating the bPDC over frequencies (Methods). On average, information flowing from the brain to the other two components was significantly higher than the inward flow (bootstrap and paired z-test, $p<0.05$ Bonferroni corrected; Fig. 2F,G).

20 Consistently, brain activity led the other two activities temporally (Fig. S3). The higher outward information

21 flow was observed for all types of movements as well (Fig. S4). Thus, the results favored Hypothesis \#3, that

22 the brain activity was mostly predictive of motor output and fluctuations of internal physiology. 
A

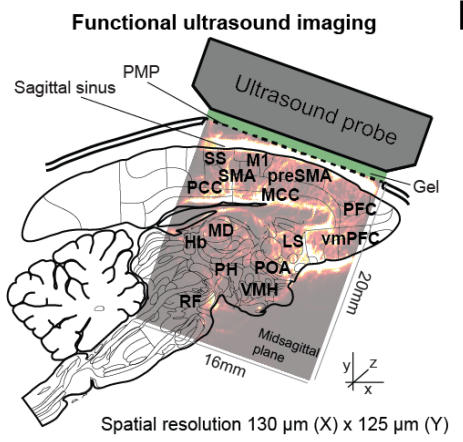

B
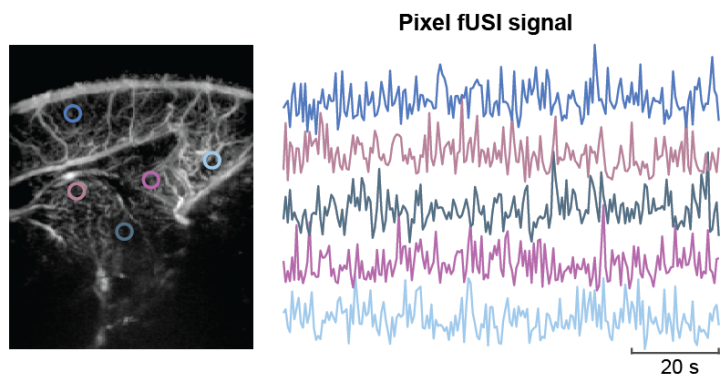

C
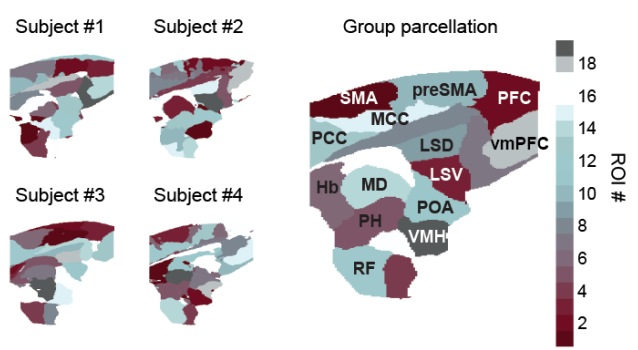

D

Block PDC

E
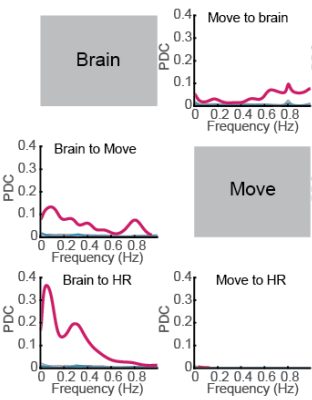

F

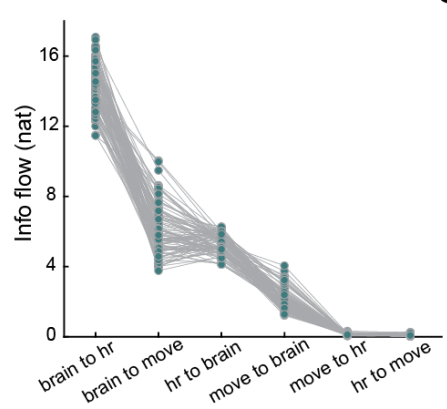

G

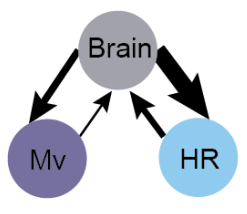

Figure 2. The brain plays an active role in coordinating movement and heart rate. (A) fUS setup and brain regions covered by imaging. (B) Exemplar pixel signals from different areas of the brain. (C) Within subject and group-level brain parcellation. We use SMA to represent the ROI covering SS, M1, and SMA. LSD: lateral septal nucleus dorsal; LSV: lateral septal nucleus ventral. (D) Illustration of bPDC. (E) bPDC of each direction. Red means significantly higher than phase-randomized surrogate distribution. Note that the lines in "HR to Move" and "Move to HR" cells are very low. (F) Estimated distribution of information flow of each direction, sorted from high to low. Outward flow from brain is greater than the corresponding inward flow ( $p<0.05$ Bonferroni corrected). (G) Information flow graph. The thickness of the arrows is proportional to information flow.

One possible mechanism coupling external and internal activities is that they could be driven by a common set of brain regions; alternatively, they could be influenced by separate regions that are themselves temporally coordinated (Fig. 3A). To test this, we first established the network composed of individual ROIs, movement, and heart rate using PDC analysis for univariate time-series (Fig. 3B) (50). The strength of connection in each direction between two nodes was quantified as integrated information flow. Two different subsets of ROIs were involved in strong information exchange with movement and heart rate (Fig. 3C, D). Thus, the temporally coordinated external and internal activities were driven by spatially separate brain regions (with the exceptions of $\mathrm{PH}$ and SMA).

We next investigated the connectivity between these two wide-spread groups of regions. One hypothesis is that the functional connectivity, defined as information flow between ROIs, exhibits two network communities corresponding to the external and internal control. Conversely, these two groups of regions might be so coupled that the network cannot be cleanly separated by the degree of participation in external and internal control. As the outward information flow was dominant, we formulated the ROI 
function as the difference between information flowing to movement and to heart rate (termed control preference). The graph revealed that ROIs with different control preference were highly interconnected and could not be separated by their functional connectivity (spectral clustering for directed graph; Fig. 3E). This suggested that the coordination between movement and heart rate was due to strongly coupled brain areas. The functional connectivity established using fUS was backed by structural connectivity from fiber tractography mapped to the same areas (functional and structural connectivity correlation $\mathrm{r}=0.46, \mathrm{p}=5.0 \times 10^{-}$ 6; Fig. S5), comparable to measurements in other modalities and species (51-53). This internally connected network ranging from the primary sensorimotor cortex for external control to the limbic areas (here mainly the LS) for internal control is consistent with the idea of 'centrifugal' brain organization (12).

A
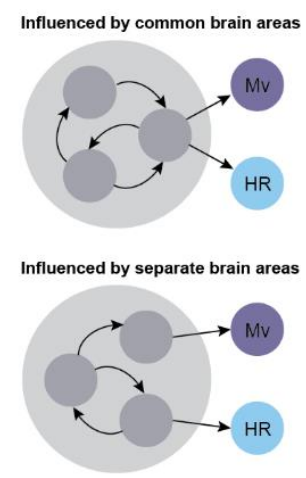

B
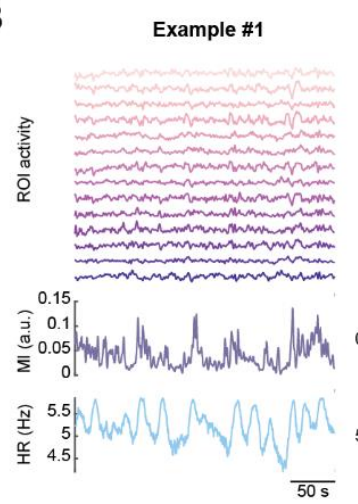
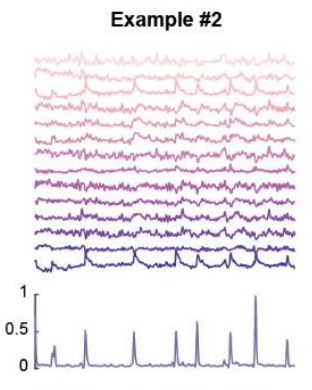
6.5
5.

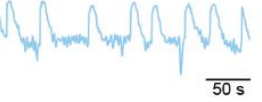

E
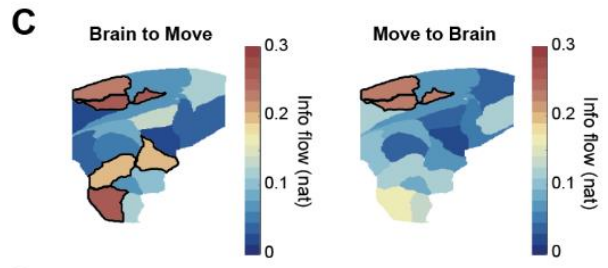

D

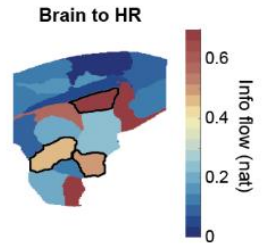

HR to Brain
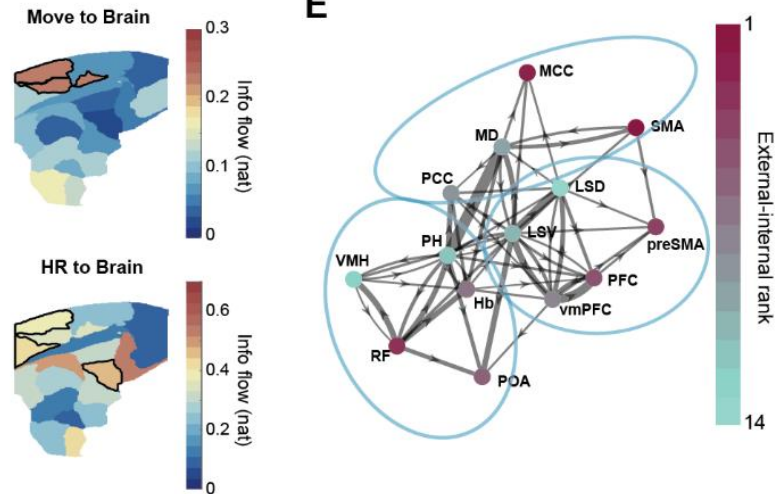

Figure 3. Separate brain regions regulate internal and external activities. (A) Hypotheses of spatial distribution for different brain areas involved in external and internal regulation. (B) Exemplars showing individual ROI signals with MI and HR. Each time series is used as a node for PDC analysis. (C, D) Brain regions sending or receiving information flow to MI and HR. Outlined areas are the ones above the 75thpercentile of the information flow within the category. (E) Brain network summary. Circled areas are communities determined by spectral clustering. Node color corresponds to the preference of control with purple towards external activity and green towards internal activity. Note that low-level subcortical nodes are separated from higher level nodes, which are further separated into two groups, but different control preferences are included in the same community.

We then investigated how the control of ongoing behavior and internal physiology were coordinated temporally. Brain regions collectively transition into different states $(38,54)$, so the regulation of external and internal activities may occur concurrently during certain states or alternatively between states. In the first case, brain regions activated during the same brain state can have different control preferences, while in the second case, areas with different control preferences are correlated with different brain states (Fig. 4A). To test these possibilities, we clustered attracting states of collective brain activity using a $2 \mathrm{D}$ embedding method described in a previous study (55) (Methods; $n=85427$ frames; Fig. 4B; Fig. S6). Four densely populated regions, representing distinct spectral and spatial activation patterns (Fig. S7), were identified in a 2D space (Fig. 4B). The fUS signals were then converted to sequences of brain states (Fig. 4C). 
We then evaluated the ROIs' participation in brain states by calculating the percentage variance of an ROI signal explained solely by the occurrence of a brain state. Most of the ROIs were significantly explained by either only states \#1 and \#4 or only states \#2 and \#3 except the MD and PFC (z-test, p<0.05; Fig. 4D). Based on this characteristic, we quantified a state score as the log-ratio between the total variance explained by $\# 1$ and $\# 4$, and by \#2 and \#3. The state score correlated significantly with control preference ( $\mathrm{r}=0.61$, $\mathrm{p}<0.05$; Fig. 4E), suggesting that external and internal regulation occurred during different brain states with \#1 and \#4 for external regulation, and \#2 and \#3 for internal regulation. Therefore, the brain alternated the control of external and internal activities.

A

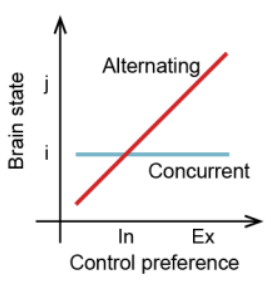

D

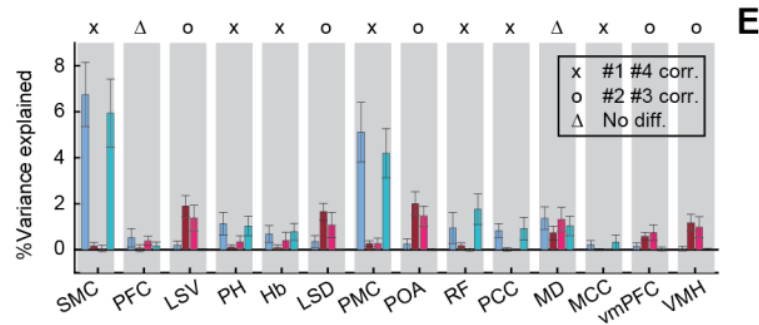

C
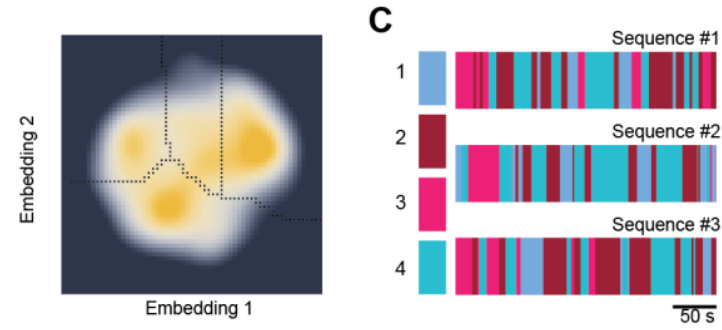

E

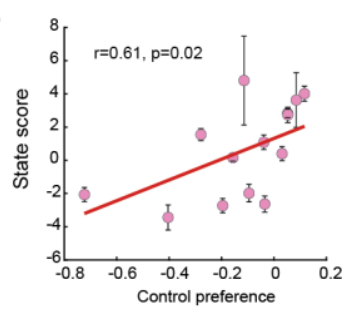

Figure 4. Brain alternates the control for external and internal activities. (A) Two possibilities for external and internal regulation relative to brain states: concurrent or alternating. (B) Watershed-based clustering on density map of t-SNE embedding space. (D) Percentage variance explained for each ROI by different brain states. Most ROIs are correlated with either \#1 \& \#4 or \#2 \& \#3. Error bars are standard deviations. (F) Correlation between state score and control preference. Error bars are standard deviations. The line is fitted by weighted linear regression.

\section{Discussion}

Our findings that brain dynamics are predictive of motor activities and heart rate fluctuations support a view of the brain as being continuously predicting external and internal activities, anticipating actions and regulatory signals $(2,3,33)$. The regulation of these two aspects is linked to spatially separate but functionally and structurally interconnected brain regions. This organization of behavior and internal physiology with brain dynamics is analogous to the blood circulatory system: Different chambers of the heart are involved in the systemic (analogous to the external interaction) and pulmonary (analogous to the internal interaction) circulations but are also synchronized by the muscle contractions of the heart. Failure of any loop will lead to the breakdown of the entire system. Although the brain is a much more complex organ, it may drive coupled interactions with the external and internal environments in a similar way. We also found that the transitions of brain states signal the switches between external and internal control. As the brain is metabolically expensive, multifunctionality in a task-free context would be less economical $(56,57)$; functional alternation might be a solution for lowering the running cost of the brain (58). Together with the predictive property of the brain, presumably on account of minimizing surprises from the external and internal environments (59), our findings suggest that the brain operates efficiently to minimize energetic cost.

Our findings do not favor the notion that brain-wide fluctuation is driven by spontaneous movements, which has been suggested by previous studies $(23,25,26)$. Information flow from brain to movement is significantly greater than the opposite direction, suggesting that the brain-wide fluctuation is 
more of an intrinsically organized pattern. Whereas there is no report in vertebrates thus far, a study in $C$. elegans observed a distributed motor command network activated even in the immobilized worm, suggesting brain-wide behavior-related activities independent of re-afferent sensory input (60). If this applies also to the vertebrates, a possible mechanism unifying the seemingly conflicting findings is that the medial brain (the focus of this study) may provide the motivation that guides behaviors $(61,62)$. This motor guidance is broadcasted to other brain areas and gets manifested in detail by interacting with local neural activities. It would be interesting to investigate the temporal coordination between the medial brain and other areas in driving spontaneous behaviors in future studies. Another reason for the seemingly different conclusion could be attributed to our different data analysis approach. Previous studies adopted multivariate linear models or cross-correlation estimating the variance explained by movements. Our adoption of PDC method allows us to distinguish direct and indirect flows in the frequency domain; this is not possible with correlation methods $(49,63)$.

Brain fluctuation correlated with physiological signals has been found universally (64-67). However, when using the hemodynamic signal as a surrogate for neural activity, such as in functional magnetic resonance imaging (fMRI) and fUS, the cardiovascular and respiratory signals have been treated as confounds and regressed out in fMRI studies (68). In addition, due to signal distortion and $\mathrm{CO}_{2}$ concentration change in the brain brought by respiration, it is also a general practice to regress out global fMRI signals approximating the removal of respiration (69). The fUS method does not have the signal distortion issue but could be influenced by $\mathrm{CO}_{2}$ concentration. Although we did not explicitly remove potential global influences in our analysis, the PDC estimate automatically treated independent external sources as noise. Thus, the global effect should be discounted at least to the extent of removing the global average. Another technical difference is that the CBV of the arterioles and capillaries measured with fUS (41) are more similar to CBV-fMRI than BOLD-fMRI (43). CBV signals exhibit a shorter onset time and time-to-peak than BOLD signals in marmosets $(70)$ and correlate linearly with neural activity for a wide range of physiological regimes (71-73). The higher sensitivity of the fUS signal may have contributed to our inference of information flow. Besides the entangled brain-physiological signal owing to the imaging technique, there is also a neurobiological basis for the correlation between brain and physiological states (64). Multiple cortical and subcortical areas participate in autonomic regulations on respiratory, cardiovascular, digestive, and other systems $(65,67,74)$. The temporal relationships between brain activity and the physiological signal are frequency dependent. For example, in mice, theta oscillations precede and are Granger-causal for the variation of respiration rate, whereas it is the opposite for the gamma oscillations (75). Our results support that at frequencies $<0.5 \mathrm{~Hz}$, the information mainly flows from brain to heart rate, outweighing the opposite direction. However, we did not exclude that heart rate and respiratory sinus arrhythmia can influence CBV at higher frequencies around blood pulsation $(\sim 5 \mathrm{~Hz})$ and respiration rate $(\sim 1 \mathrm{~Hz})$ in marmosets.

Intrinsic functional architecture revealed in slow spontaneous fluctuations across the brain is not unique to humans (70) and has been observed across various animal species (77-82). With technologies allowing for higher spatial and temporal resolutions, we are now able to better understand the neural and hemodynamic accounts for behavior on a large scale (83). Using fUS, we revealed how heart rate, a measure of the moment-to-moment variation of energy supply to the body, is coordinated with motor activity, which consumes this energy - via a complex brain network. The brain is at the nexus between internal and external environments. Its functional division into external and internal regulations, and the inseparable nature of these two dynamics, support a view of the default mode network as a dynamic sense-making network that actively integrates external and internal life events (84). This coordination may be a consequence of contextdependent energy optimization. Questions for future studies are how the brain exploits and reconfigures its intrinsic networks in the face of different internal and external challenges in contexts such as social interaction and learning $(85,86)$, and how life experiences can shape the energy landscape of network dynamics and give rise to the diversity of behavioral phenotypes (84). 
Methods

Subjects

All experiments were performed in compliance with the Princeton University Institutional Animal Care and Use Committee guidelines. The subjects used in the study were four adult common marmosets $(>2$ years old, one female and three males) housed at Princeton University. All four subjects participated in the fUS recording; three of them were also used for ECG and video recording. The colony room was maintained at $27^{\circ} \mathrm{C}$ temperature and 50-60\% humidity with a 12L:12D light cycle. Marmosets had ad libitum access to water and were fed with a standard diet. All subjects were acclimated to the experimental environment and positioning at least a month before the formal experiments.

Surgery

For pre-operative procedures, the animal was placed on a warmed blanket with temperature, pulse, respiration and SPO2 being monitored and blood glucose measured. Dexamethasone $1 \mathrm{mg} / \mathrm{kg}$ and Baytril 5 $\mathrm{mg} / \mathrm{kg}$ was administered IM. The animal was induced with alfaxalone $10 \mathrm{mg} / \mathrm{kg}$ IM before intubation. The intubated animal was then connected to the anesthesia machine and moved into the OR. The surgical site was clipped, and the remaining hair was removed with Nair. The surgical site was cleaned with betadine and covered with surgical drape (3M). Didocaine was injected into the scalp at multiple sites. An incision ( 20 $\mathrm{mm}$, rostral/caudal orientation) was made to expose the skull along the top of the head through the scalp. Tissue was reflected with a retractor to expose the skull. Sterilized miniature titanium screws were inserted into the bone at several locations to anchor the head post and the head plate. A customized head post and a head plate were immobilized on the skull with adhesive cement (C\&B Metabond Quick Adhesive Cement System, Parkell). The head plate allowed the ultrasound probe to be aligned with the midline of the brain. Within the head plate, a cranial window of $\sim 8 \mathrm{~mm} \times 16 \mathrm{~mm}$ was created. During off-experiment time, a stainless-steel cover was attached to the head plate to protect the head plate and the craniotomy.

\section{Functional ultrasound (fUS) imaging}

Our custom ultrasound probe allowed a wide field of view (20 mm depth, $16 \mathrm{~mm}$ width), a temporal resolution of $2 \mathrm{~Hz}$, a spatial resolution of $125 \mu \mathrm{m}$ in width, $130 \mu \mathrm{m}$ in depth, and $200-800 \mu \mathrm{m}$ in thickness depending on the depth (200 $\mu \mathrm{m}$ at $12 \mathrm{~mm}$ depth). The probe was connected to an ultrasound scanner (Vantage 128, Verasonics) controlled by a PC. fUS signals were acquired at the midline sagittal plane. The image acquisition method was described previously (43).

\section{Experimental protocol for fUS recording}

Each subject participated in experiments once per day around the same time of the day. The subject was brought to the experimental room from their home cage. The walls of the room $(3.2 \mathrm{~m} \times 5.5 \mathrm{~m})$ were covered with sound attenuating foam. The subject was placed in a custom-designed partial restraint device with the head fixed by the head post. The cover was removed to expose the cranial window, which was then flushed with sterile water and covered by sterile ultrasound gel (Sterile Aquasonic 100 Ultrasound Gel). A customized probe holder was then attached to the top of the head plate with screws and the ultrasound probe was placed inside the holder. The head post was released before the experiment to allow head movement. An initial image was acquired to examine the imaging position and quality. Each task-absent trial lasted 10-20 min. After the experiment, the recording surface was cleaned with $0.05 \%$ chlorhexidine and sterile water and was closed with the cover. The animal was then returned to their home cage.

\section{Image processing}

We first aligned image frames using a rigid body transformation within each session to eliminate any slow drift of image position within the FOV. To achieve this, we first calculated an averaged image across the session and saved it as a reference. Then we aligned each frame to this reference using elastix software (87). To align all sessions to a common reference, we next consecutively performed rigid body, affine, and B-spline transformations using elastix. We then created a mask for the within brain area shared across all sessions with the sagittal sinus excluded. 
To remove timepoints contaminated by motion artifacts, we used the averaged signal of an area outside the brain as control. We set a criterion for a timepoint to be an outlier if the control signal was above 1.5(Q3-Q1)+Q3, where Qi stands for the $i^{\text {th }}$ quartile of the control signal. Once the noise was removed, we band-pass filtered the image data between $0.005 \mathrm{~Hz}$ and $0.5 \mathrm{~Hz}$ and standardized the signal of each pixel. Finally, we spatially smoothed the images using a 2D Gaussian filter with sigma=2 pixels in each dimension.

\section{Brain parcellation}

We first vectorized the image data such that each column was the masked pixels within a frame. To perform group parcellation across all sessions, we calculated principal component analysis (PCA) to reduce the temporal dimensionality (\# of columns) to 30 for each session and concatenated all sessions by the reduced dimension (88). We then calculated the pairwise Spearman's rank correlation coefficient, based on which we established a distance matrix $A$ with diagonal elements 0 and off-diagonals $A_{i j}=\left|\rho_{i j}\right| \sqrt{\frac{n-2}{1-\rho_{i j}^{2}}}$, where $n$ the number of pixels, $\rho_{i j}$ the Spearman's correlation between pixel $i$ and $j$. We then performed spectral clustering (89) using the distance matrix for which we calculated the symmetric normalized Laplacian matrix $L=D^{-\frac{1}{2}} A D^{-\frac{1}{2}}$, where $D_{i i}=\sum_{j} A_{i j}$ the degree matrix. We calculated the eigenvectors corresponding to the largest $k$ eigenvalues of $L$ and performed k-means clustering on the eigenvectors. This yields the spectral clustering results. Similar procedure was carried out for the subject specific parcellation.

\section{Cardiac data acquisition}

To record ECG, we put on an elastic band to the chest of the marmosets with a pair of $\mathrm{Ag}$-AgCl surface electrodes (Grass Technology) sewed on. The data was resampled into $1 \mathrm{kHz}$ and band-pass filtered between 15 and $100 \mathrm{~Hz}$. To identify heartbeats, we calculated sliding correlation coefficients between session-specific templates and the ECG signal, and these locations were marked as one if the correlation coefficient was above a threshold. This binary signal was convolved with a Gaussian window to estimate the momentary heart rate.

\section{Behavioral videography}

In a lighted room, the subject was placed in a customized chair with its front facing a camera (Logitech C920). Videos of the subjects were recorded at 24-Hz framerate and synchronized with fUS acquisition. Essentially, a $1 \mu$ s pulse signaling the end of image acquisition ( $400 \mathrm{~ms}$ long per $500 \mathrm{~ms}$ cycle) was passed to the interrupt channel of an Arduino board, which converted the signal to a $200 \mu$ s TTL pulse to be recorded. The sound was also recorded from a microphone placed next to the subject (Zoom H4n Pro). Audio, TTL pulse, and ECG signal were simultaneously recorded through a data acquisition box (Muscle SpikerBox Pro) at a sample rate of $44.1 \mathrm{kHz}$. This audio signal was aligned with the audio channel of the video through a brief playback of white noise. We created masks for the subject based on pixel variance contrasting the static background and aligned the masked images across sessions through rigid body transformation and affine transformation using elastix. Video analysis was carried out for the aligned videos. We defined motion intensity (MI) as the absolute value of the total intensity difference between two adjacent frames, scaled by the mean across the session. We then downsampled MI to $2 \mathrm{~Hz}$.

To extract different types of motor behaviors, we performed a group independent component analysis (ICA). First, we carried out group PCA by concatenating the first 100 spatial PCs across 35 video sessions. Next, we performed ICA on the group PCs and identified 34 spatial modes. By inspecting the spatial patterns, we categorized the behaviors into six types: blink, mouth movement, ear movement, arm and hand movement, foot and tail movement, and body movement. We calculated the momentary behavioral index as the inner product between each IC and the video frame, then took the maximal value at each time point across the subset of ICs belonging to the same behavioral category. To estimate the fractional time spent on each type of behavior, we calculated the proportion of time during which behavior indices were above a threshold of the session. Time not spent on any movements was determined as being still. 


\section{Coberence}

The coherence between motion intensity and heart rate was calculated using the MATLAB function $\mathrm{cm} t m$. We concatenated the time series of all 35 sessions to minimize the edge effect. To remove the baseline difference in heart rate by sessions, we used the $z$-score of heart rate. Statistical significance was determined as being greater than the $95 \%$ CI of the null coherence, which was estimated by phase-randomized surrogates of the time series repeated 1000 times. We separated periods of substantial movements and subtle movements using a threshold of MI. If a substantial movement was detected, we also included the timepoints $10 \mathrm{~s}$ before and after. The rest was considered to contain only subtle movements. The coherence analyses were repeated for concatenated segments of substantial and subtle movements.

\section{Partial directed coherence}

We used partial directed coherence (PDC) to elucidate the coordination between brain and peripheral activities. To combine time-series across all sessions, we normalized ROI signals and HR, but MI, within each session. By concatenating the time series, we reduced potential edge effects and increased statistical robustness. We treated the ROIs as multivariate time-series and applied block-PDC (bPDC) for the total causality measure between the brain and peripheral components. The bPDC as an extension to PDC is available at https://www.lcs.poli.usp.br/ baccala/pdc/canon/. We designed three blocks of time series, consisting of 1) all ROIs, 2) MI, 3) HR. Here we used all ROIs, including those identified as big vessels. The optimal maximum order of the autoregressive model was determined using the Akaike information criteria (AIC). To find significantly high PDC, we phase-randomized the time-series 1000 times and calculated the $95 \%$ CI of the null distribution. We set the criteria for statistical significance with Bonferroni correction for multiple comparisons $\alpha=0.05 / 6$, as there were 6 directional relationships to examine. For PDC of univariate time-series, we applied the package available at https://www.lcs.poli.usp.br/ $\sim \mathrm{baccala} / \mathrm{pdc} /$. The maximum order of the autoregressive model was determined using AIC.

We adopted the informational metric of PDC $\left|\iota \pi_{i j}(\omega)\right|^{2}$, which is essentially linked to the mutual information rate via $M I R_{i j}=-\frac{1}{4 \pi} \int_{-\pi}^{\pi} \log \left(1-\left|\iota \pi_{i j}(\omega)\right|^{2}\right) d \omega(50)$. In this work, we used a similar measure as MIR for total information flow $I_{i j}=-\int_{0}^{f_{c}} \log \left(1-\left|\iota \pi_{i j}(f)\right|^{2}\right) d f$, where the cutoff frequency $f_{c}=0.5$ Hz. To test the difference between $I_{i_{1} j_{1}}$ and $I_{i_{2} j_{2}}$, we bootstrapped the data by resampling sessions with replacement and calculated bPDC for each resampled time series. This procedure was repeated 1000 times to estimate the distribution of information flow in each direction. We then compared each pair of directions using paired z-test with a Bonferroni corrected criterion $\alpha=0.05 / 15$.

We defined information flow above the 75th-percentile within each category as strongly influencing directions. The categories were 1) $I_{i j}$ for $\left.i, j \in R O I s, 2\right) I_{i j}$ for $i \in R O I s, j=M I$ and $i=M I, j \in R O I s$, and 3) $I_{i j}$ for $i \in R O I s, j=H R$ and $i=H R, j \in R O I s$.

\section{Directed graph clustering}

We adopted the weighted cuts algorithm for spectral clustering in a directed graph (90). The MATLAB package can be found at https://sites.stat.washington.edu/mmp/software.html. Essentially, we normalized the Laplacian matrix with node weights $T_{i}$, here we used $T_{i}=D_{i}=\sum_{j} A_{i j}$, which is simply the out-degree. We next found the eigenvectors corresponding to the $k$ smallest eigenvalues of $H=\frac{1}{2} T^{-\frac{1}{2}}(2 D-A-$ $\left.A^{T}\right) T^{-\frac{1}{2}}$, and then performed the k-means clustering on the eigenvectors.

\section{Brain state clustering}

We followed (55) to decompose imaging data into a spectro-spatial space. We calculated 25 group independent components (group-ICA) representing the spatial distribution of potential source signals. The group-ICA was performed based on the temporally reduced concatenated data described above for spectral clustering (88). We filtered the brain images into spectral bands using maximal overlap discrete wavelet packet transformation with a Daubechies wavelet of 2 vanishing moments. Then we multiplied the ICs and each of 
the frequency bands of the brain image $\tilde{X}_{f}=S W_{f}$, where $S$ the ICs of size $n_{i c} \times n_{\text {pixel }}$ and $W_{f}$ the wavelet packet transform of frequency $f$ with size of $n_{\text {pixel }} \times n_{\text {time }}$. Thus, the dimension of a time point was reduced to $n_{\text {ic }} \times n_{\text {freq }}=400$.

One problem with group-level clustering is that variations created by experiments due to slight differences in, e.g., probe positioning and signal quality and the difference in subjects may become dominant and drive the clustering results. To deal with this, we minimized the dependence on experimental sessions using an expectation-maximization (EM) procedure with penalization on session dependence in the objective function of $\mathrm{K}$-means clustering. Then, we transformed the data iteratively to a common space across sessions. This algorithm was based on the Harmony method developed for high-dimensional biological data like RNA-seq (91). In this study, we modified this algorithm to deal with time series with the temporal structure maintained during the optimization by applying fused lasso regularization (the elaborated method will appear in a separate article). The transformed data yielded clustering with session-dependence largely reduced (Fig. S7). Data used for the correction were the dimensionality reduced data described above.

We further performed manifold embedding onto a $2 \mathrm{D}$ space for the corrected data using the $\mathrm{t}$ distributed stochastic neighbor embedding (t-SNE) algorithm. MATLAB routine tsne was used with the Barnes-hut approximations, correlational distances, and perplexity of 32. Following (55), we performed a watershed transform of the negative density map to identify densely populated regions. The density map was constructed from a 2D histogram with a Gaussian filter. The width of the Gaussian filter controlled the granularity of the density map. We chose the parameters to ensure that each cluster across multiple sessions was not significantly biased by a single trial. The watershed transform was performed using the MATLAB function watershed.

\section{State score}

To evaluate to what extend the ROI activity was attributed to changes in brain state, we estimated the percentage variance of ROI activity explained by the occurrence of each brain state using cross-validation. We randomly stratified the data into ten folds, trained linear models of $y_{i}=\alpha_{i j}+\beta_{i j} x_{j}+\epsilon_{i}$ where $y_{i}$ the activity of the ith ROI, $x_{j}$ the one-hot encoding of brain state $j$, and $\epsilon_{i}$ a random noise, using the nine folds of the data, and calculated test variance explained as $R^{2}=1-R S S / T S S$ using the remaining fold. We estimated the mean and standard deviation of variance explained across the 10-time cross-validations for each ROI and each brain state. Assuming the variances explained were normally distributed, we tested whether the variance explained was equal to zero. The null hypothesis would be rejected if the mean variance explained was greater than 1.96 times the estimated standard deviation. Our results showed that states \#1 and \#4 covaried and \#2 and \#3 co-varied. We thus defined a state score as $S S_{i}=\log \left(\widehat{R}_{i 1}^{2}+\hat{R}_{i 4}^{2}\right)-\log \left(\hat{R}_{i 2}^{2}+\widehat{R}_{i 3}^{2}\right)$, where $\hat{R}_{i j}^{2}$ is the variance explained for ROI \#i by the $\mathrm{j}$-th state. Thus, if a signal were more strongly correlated with \#1 and \#4 than \#2 and \#3, the state score would be positive, and vice versa. To estimate the variance of the state scores, we applied chain rule $\hat{\sigma}_{S S_{i}}^{2}=\frac{\widehat{\sigma}_{i 1}^{2}+\widehat{\sigma}_{i 4}^{2}}{\left(\hat{R}_{i 1}^{2}+\hat{R}_{i 4}^{2}\right)^{2}}+\frac{\widehat{\sigma}_{i 2}^{2}+\widehat{\sigma}_{i 3}^{2}}{\left(\hat{R}_{i 2}^{2}+\widehat{R}_{i 3}^{2}\right)^{2}}$.

\section{State correlation with control preference}

We defined the control preference as the difference between the information flow from ROI to movement and from ROI to heart rate: $C P_{i}=I_{i \rightarrow \text { move }}-I_{i \rightarrow H R}$. To test the dependence of control preference on brain state, using each ROI as a sample, we performed a weighted linear regression with the weight as $1 / \hat{\sigma}_{s s_{i}}^{2}$ (a similar result was obtained with ordinary least squares). The criterion for statistical significance was set at $\alpha=$ 0.05 . 


\section{Reference}

1. W. B. Cannon, Organization for Physiological Homeostasis. Physiological reviews 9, 399-431 (1929).

2. G. Pezzulo, F. Rigoli, K. Friston, Active Inference, homeostatic regulation and adaptive behavioural control. Progress in Neurobiology 134, 17-35 (2015).

3. A. K. Seth, Interoceptive inference, emotion, and the embodied self. Trends in cognitive sciences 17, 565-573 (2013).

4. D. J. Anderson, Circuit modules linking internal states and social behaviour in flies and mice. Nature Reviews Neuroscience 17, 692-704 (2016).

5. M. A. Gray et al., Emotional appraisal is influenced by cardiac afferent information. Emotion 12, 180-191 (2012).

6. Y. S. Zhang, A. A. Ghazanfar, Perinatally Influenced Autonomic System Fluctuations Drive Infant Vocal Sequences. Current biology: $C B$, (2016).

7. J. I. Borjon, D. Y. Takahashi, D. C. Cervantes, A. A. Ghazanfar, Arousal dynamics drive vocal production in marmoset monkeys. Journal of neurophysiology 116, 753-764 (2016).

8. D. A. Liao, Y. S. Zhang, L. X. Cai, A. A. Ghazanfar, Internal states and extrinsic factors both determine monkey vocal production. Proceedings of the National Academy of Sciences of the United States of America 115, 3978-3983 (2018).

9. S. Skora, F. Mende, M. Zimmer, Energy Scarcity Promotes a Brain-wide Sleep State Modulated by Insulin Signaling in C. elegans. Cell Reports 22, 953-966 (2018).

10. D. S. Margulies et al., Situating the default-mode network along a principal gradient of macroscale cortical organization. Proceedings of the National Academy of Sciences 113, 1257412579 (2016).

11. J. M. Huntenburg, P.-L. Bazin, D. S. Margulies, Large-Scale Gradients in Human Cortical Organization. Trends in cognitive sciences 22, 21-31 (2018).

12. M. Mesulam, From sensation to cognition. Brain 121, 1013-1052 (1998).

13. O. Pollatos, R. Schandry, D. P. Auer, C. Kaufmann, Brain structures mediating cardiovascular arousal and interoceptive awareness. Brain research 1141, 178-187 (2007).

14. J. R. Andrews-Hanna, The Brain's Default Network and Its Adaptive Role in Internal Mentation. The Neuroscientist 18, 251-270 (2011).

15. A. G. Obukhov et al., Differences in Human Cortical Gene Expression Match the Temporal Properties of Large-Scale Functional Networks. PloS one 9, e115913 (2014).

16. Y. Golland, P. Golland, S. Bentin, R. Malach, Data-driven clustering reveals a fundamental subdivision of the human cortex into two global systems. Neuropsychologia 46, 540-553 (2008).

17. M. Boly et al., Baseline brain activity fluctuations predict somatosensory perception in humans. Proceedings of the National Academy of Sciences 104, 12187-12192 (2007).

18. M. Vinck, R. Batista-Brito, U. Knoblich, Jessica A. Cardin, Arousal and Locomotion Make Distinct Contributions to Cortical Activity Patterns and Visual Encoding. Neuron 86, 740-754 (2015).

19. C. M. Niell, M. P. Stryker, Modulation of Visual Responses by Behavioral State in Mouse Visual Cortex. Neuron 65, 472-479 (2010).

20. A. R. Damasio et al., Subcortical and cortical brain activity during the feeling of self-generated emotions. Nature Neuroscience 3, 1049-1056 (2000).

21. J. W. de Gee et al., Dynamic modulation of decision biases by brainstem arousal systems. eLife 6, (2017).

22. W. E. Allen et al., Thirst regulates motivated behavior through modulation of brainwide neural population dynamics. Science 364, (2019). 
23. C. Stringer et al., Spontaneous behaviors drive multidimensional, brainwide activity. Science 364, eaav7893 (2019).

24. P. J. Drew, A. T. Winder, Q. Zhang, Twitches, Blinks, and Fidgets: Important Generators of Ongoing Neural Activity. The Neuroscientist 25, 298-313 (2018).

25. S. Musall, M. T. Kaufman, A. L. Juavinett, S. Gluf, A. K. Churchland, Single-trial neural dynamics are dominated by richly varied movements. Nature Neuroscience 22, 1677-1686 (2019).

26. D. A. McCormick, E. McCarthy, E. Zagha, D. B. Salkoff, Movement and Performance Explain Widespread Cortical Activity in a Visual Detection Task. Cerebral Cortex 30, 421-437 (2020).

27. H. S. Kaplan, M. Zimmer, Brain-wide representations of ongoing behavior: a universal principle? Current opinion in neurobiology 64, 60-69 (2020).

28. A. Damasio, G. B. Carvalho, The nature of feelings: evolutionary and neurobiological origins. Nature Reviews Neuroscience 14, 143-152 (2013).

29. Matthew J. McGinley et al., Waking State: Rapid Variations Modulate Neural and Behavioral Responses. Neuron 87, 1143-1161 (2015).

30. R. V. Raut et al., Global waves synchronize the brain's functional systems with fluctuating arousal. Science Advances 7, eabf2709 (2021).

31. R. A. Adams, S. Shipp, K. J. Friston, Predictions not commands: active inference in the motor system. Brain Structure and Function 218, 611-643 (2012).

32. G. B. Keller, T. D. Mrsic-Flogel, Predictive Processing: A Canonical Cortical Computation. Neuron 100, 424-435 (2018).

33. L. F. Barrett, W. K. Simmons, Interoceptive predictions in the brain. Nature Reviews Neuroscience 16, 419-429 (2015).

34. P. Sterling, Allostasis: A model of predictive regulation. Physiology \& behavior 106, 5-15 (2012).

35. A. K. Seth, K. J. Friston, Active interoceptive inference and the emotional brain. Philosophical Transactions of the Royal Society B: Biological Sciences 371, 20160007 (2016).

36. A. T. Winder, C. Echagarruga, Q. Zhang, P. J. Drew, Weak correlations between hemodynamic signals and ongoing neural activity during the resting state. Nature Neuroscience 20, 1761-1769 (2017).

37. C. Julien, The enigma of Mayer waves: Facts and models. Cardiovascular research 70, 12-21 (2006).

38. D. Gutierrez-Barragan, M. A. Basson, S. Panzeri, A. Gozzi, Infraslow State Fluctuations Govern Spontaneous fMRI Network Dynamics. Current Biology 29, 2295-2306.e2295 (2019).

39. J. M. Palva, S. Palva, Infra-slow fluctuations in electrophysiological recordings, bloodoxygenation-level-dependent signals, and psychophysical time series. Neurolmage 62, 22012211 (2012).

40. P. J. Drew, C. Mateo, K. L. Turner, X. Yu, D. Kleinfeld, Ultra-slow Oscillations in fMRI and RestingState Connectivity: Neuronal and Vascular Contributions and Technical Confounds. Neuron 107, 782-804 (2020).

41. E. Macé et al., Functional ultrasound imaging of the brain. Nature Methods 8, 662-664 (2011).

42. A. Urban et al., Real-time imaging of brain activity in freely moving rats using functional ultrasound. Nature Methods 12, 873-878 (2015).

43. É. Macé et al., Whole-Brain Functional Ultrasound Imaging Reveals Brain Modules for Visuomotor Integration. Neuron 100, 1241-1251.e1247 (2018).

44. M. E. Raichle, The Brain's Default Mode Network. Annual review of neuroscience 38, 433-447 (2015).

45. V. Napadow et al., Brain correlates of autonomic modulation: Combining heart rate variability with fMRI. Neurolmage 42, 169-177 (2008).

46. A. Woodward et al., The Brain/MINDS 3D digital marmoset brain atlas. Scientific Data 5, (2018). 
47. C. D. Hardman, K. W. Ashwell, Stereotaxic and chemoarchitectural atlas of the brain of the common marmoset (Callithrix jacchus). (CRC press, 2012).

48. D. Y. Takahashi, L. A. Baccalá, K. Sameshima, Canonical information flow decomposition among neural structure subsets. Frontiers in Neuroinformatics 8, (2014).

49. L. A. Baccalá, K. Sameshima, Partial directed coherence: a new concept in neural structure determination. Biol Cybern 84, 463-474 (2001).

50. D. Y. Takahashi, L. A. Baccalá, K. Sameshima, Information theoretic interpretation of frequency domain connectivity measures. Biol Cybern 103, 463-469 (2010).

51. A. Díaz-Parra, Z. Osborn, S. Canals, D. Moratal, O. Sporns, Structural and functional, empirical and modeled connectivity in the cerebral cortex of the rat. Neurolmage 159, 170-184 (2017).

52. Y. Hori et al., Comparison of resting-state functional connectivity in marmosets with tracerbased cellular connectivity. Neurolmage 204, 116241 (2020).

53. A. Messé, Parcellation influence on the connectivity-based structure-function relationship in the human brain. Human Brain Mapping 41, 1167-1180 (2019).

54. C. Chang, G. H. Glover, Time-frequency dynamics of resting-state brain connectivity measured with fMRI. Neuroimage 50, 81-98 (2010).

55. J. C. W. Billings et al., Instantaneous brain dynamics mapped to a continuous state space. Neurolmage 162, 344-352 (2017).

56. J. W. Rocks, H. Ronellenfitsch, A. J. Liu, S. R. Nagel, E. Katifori, Limits of multifunctionality in tunable networks. Proceedings of the National Academy of Sciences 116, 2506-2511 (2019).

57. E. Bullmore, O. Sporns, The economy of brain network organization. Nature Reviews Neuroscience 13, 336-349 (2012).

58. D. Attwell, S. B. Laughlin, An Energy Budget for Signaling in the Grey Matter of the Brain. Journal of Cerebral Blood Flow \& Metabolism 21, 1133-1145 (2016).

59. K. Friston, The free-energy principle: a unified brain theory? Nature Reviews Neuroscience 11, 127-138 (2010).

60. S. Kato et al., Global Brain Dynamics Embed the Motor Command Sequence of Caenorhabditis elegans. Cell 163, 656-669 (2015).

61. D. Stawarczyk, M. A. Bezdek, J. M. Zacks, Event Representations and Predictive Processing: The Role of the Midline Default Network Core. Topics in Cognitive Science 13, 164-186 (2019).

62. M. E. Raichle, D. A. Gusnard, Intrinsic brain activity sets the stage for expression of motivated behavior. The Journal of comparative neurology 493, 167-176 (2005).

63. L. A. Baccalá, K. Sameshima, Chapter 3 Overcoming the limitations of correlation analysis for many simultaneously processed neural structures. 130, 33-47 (2001).

64. C. Chang et al., Association between heart rate variability and fluctuations in resting-state functional connectivity. Neurolmage 68, 93-104 (2013).

65. I. Rebollo, A.-D. Devauchelle, B. Béranger, C. Tallon-Baudry, Stomach-brain synchrony reveals a novel, delayed-connectivity resting-state network in humans. eLife 7, (2018).

66. K. Kim et al., Resting-State Neural Firing Rate Is Linked to Cardiac-Cycle Duration in the Human Cingulate and Parahippocampal Cortices. The Journal of Neuroscience 39, 3676-3686 (2019).

67. F. Beissner, K. Meissner, K. J. Bar, V. Napadow, The Autonomic Brain: An Activation Likelihood Estimation Meta-Analysis for Central Processing of Autonomic Function. Journal of Neuroscience 33, 10503-10511 (2013).

68. K. Murphy, R. M. Birn, P. A. Bandettini, Resting-state fMRI confounds and cleanup. Neurolmage 80, 349-359 (2013).

69. J. D. Power et al., Ridding fMRI data of motion-related influences: Removal of signals with distinct spatial and physical bases in multiecho data. Proceedings of the National Academy of Sciences 115, E2105-E2114 (2018). 
70. Y. Hirano et al., Investigation of the BOLD and CBV fMRI responses to somatosensory stimulation in awake marmosets (Callithrix jacchus). NMR in Biomedicine 31, e3864 (2018).

71. A. O. Nunez-Elizalde et al., Neural basis of functional ultrasound signals. (2021).

72. A.-K. Aydin et al., Transfer functions linking neural calcium to single voxel functional ultrasound signal. Nature communications 11, (2020).

73. D. Boido et al., Mesoscopic and microscopic imaging of sensory responses in the same animal. Nature communications 10, (2019).

74. D. F. Cechetto, Cortical control of the autonomic nervous system. Experimental Physiology 99, 326-331 (2014).

75. A. B. L. Tort, M. Hammer, J. Zhang, J. Brankačk, A. Draguhn, Temporal Relations between Cortical Network Oscillations and Breathing Frequency during REM Sleep. The Journal of Neuroscience 41, 5229-5242 (2021).

76. M. D. Fox, M. E. Raichle, Spontaneous fluctuations in brain activity observed with functional magnetic resonance imaging. Nature Reviews Neuroscience 8, 700-711 (2007).

77. J. M. Li, W. J. Bentley, L. H. Snyder, Functional connectivity arises from a slow rhythmic mechanism. Proceedings of the National Academy of Sciences 112, E2527-E2535 (2015).

78. M. P. Vanni, A. W. Chan, M. Balbi, G. Silasi, T. H. Murphy, Mesoscale Mapping of Mouse Cortex Reveals Frequency-Dependent Cycling between Distinct Macroscale Functional Modules. The Journal of Neuroscience 37, 7513-7533 (2017).

79. A. Mitra et al., Spontaneous Infra-slow Brain Activity Has Unique Spatiotemporal Dynamics and Laminar Structure. Neuron 98, 297-305.e296 (2018).

80. A. M. Belcher et al., Large-Scale Brain Networks in the Awake, Truly Resting Marmoset Monkey. Journal of Neuroscience 33, 16796-16804 (2013).

81. M. P. Schroeder, C. Weiss, D. Procissi, J. F. Disterhoft, L. Wang, Intrinsic connectivity of neural networks in the awake rabbit. Neurolmage 129, 260-267 (2016).

82. C. P. Pawela et al., Resting-state functional connectivity of the rat brain. Magnetic Resonance in Medicine 59, 1021-1029 (2008).

83. P. Pais-Roldán et al., Contribution of animal models toward understanding resting state functional connectivity. Neurolmage 245, 118630 (2021).

84. Y. Yeshurun, M. Nguyen, U. Hasson, The default mode network: where the idiosyncratic self meets the shared social world. Nature Reviews Neuroscience 22, 181-192 (2021).

85. D. S. Bassett et al., Dynamic reconfiguration of human brain networks during learning. Proceedings of the National Academy of Sciences 108, 7641-7646 (2011).

86. R. Schmälzle et al., Brain connectivity dynamics during social interaction reflect social network structure. Proceedings of the National Academy of Sciences 114, 5153-5158 (2017).

87. S. Klein, M. Staring, K. Murphy, M. A. Viergever, J. Pluim, elastix: A Toolbox for Intensity-Based Medical Image Registration. IEEE Transactions on Medical Imaging 29, 196-205 (2010).

88. S. M. Smith, A. Hyvärinen, G. Varoquaux, K. L. Miller, C. F. Beckmann, Group-PCA for very large fMRI datasets. Neurolmage 101, 738-749 (2014).

89. A. Y. Ng, M. I. Jordan, Y. Weiss, in Advances in neural information processing systems. (2002), pp. 849-856.

90. M. Meilă, W. Pentney, Clustering by weighted cuts in directed graphs. 135-144 (2007).

91. I. Korsunsky et al., Fast, sensitive and accurate integration of single-cell data with Harmony. Nature Methods 16, 1289-1296 (2019). 


\section{Acknowledgments}

2 We thank Uri Hasson and Samuel Nastase for comments on the manuscript. This work was supported by an 3 NIH-NINDS grant to A.A.G. (R01NS054898).

4

6 Conceptualization: YSZ, AAG

7 Methodology: YSZ, DYT

8 Funding acquisition: $\mathrm{AAG}$

9 Data collection: DYT, AEH, YSZ, DAL

10 Data analysis: YSZ

11 Writing: YSZ, AAG

12

13

14

Data and material availability:

Data is available from Dryad Digital Repository doi:10.5061/dryad.jwstqjq9x 


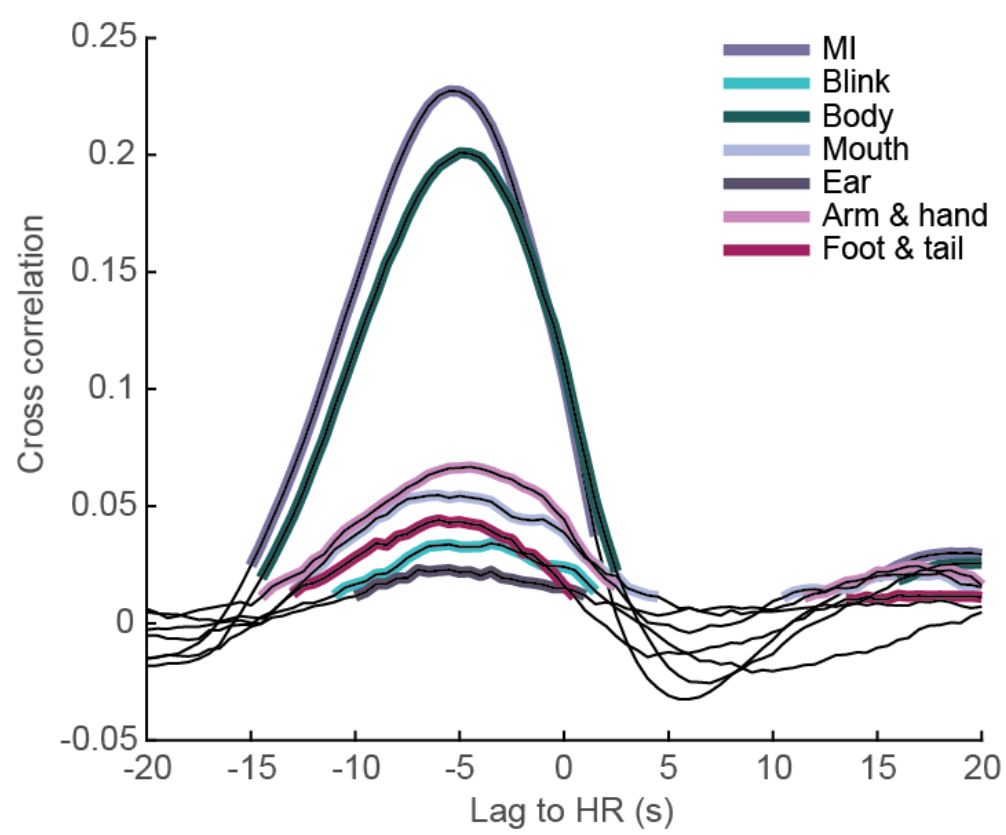

Fig. S1. Cross correlation between HR and different types of movements and MI. Highlighted segments are significant against the $95 \%$ CI of phase randomized data. Positive lag is HR leading and vice versa. The time lags of different movements and MI are consistent.

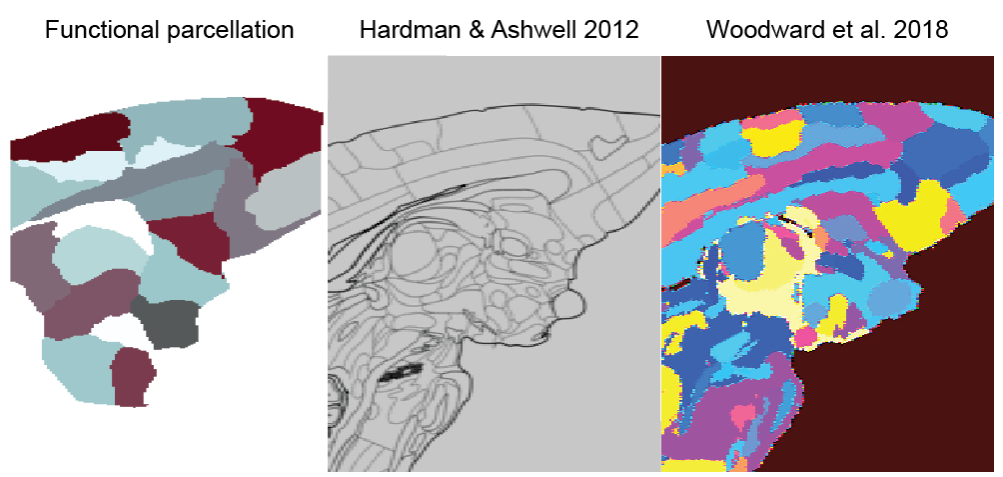

Fig. S2. Functional parcellation compared with two marmoset brain atlases. 


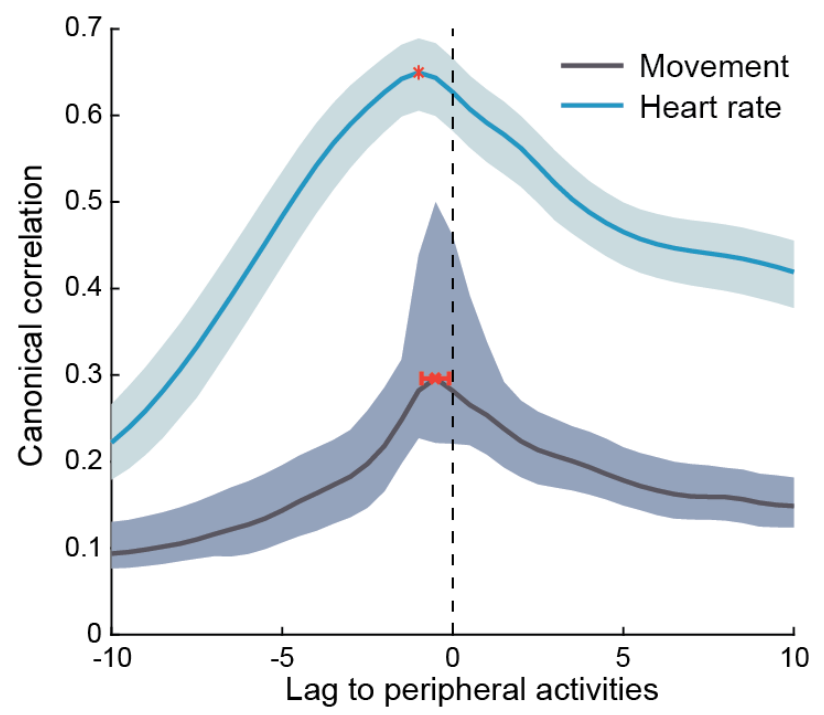

Fig. S3. Canonical correlations between ROI activities and movement and between ROI activities and heart rate. Shaded areas are $95 \%$ CI. Red bars label the peak position. For movement, the lag of brain is $-0.51 \pm 0.40 \mathrm{~s}$, and for heart rate it is $-1.0 \pm 0.0 \mathrm{~s}$ (mean $+\backslash$ - s.d.). A negative lag means that brain is leading.

\section{Brain to blink}

Brain to body

Brain to mouth

Brain to ear

Brain to arm

Brain to foot
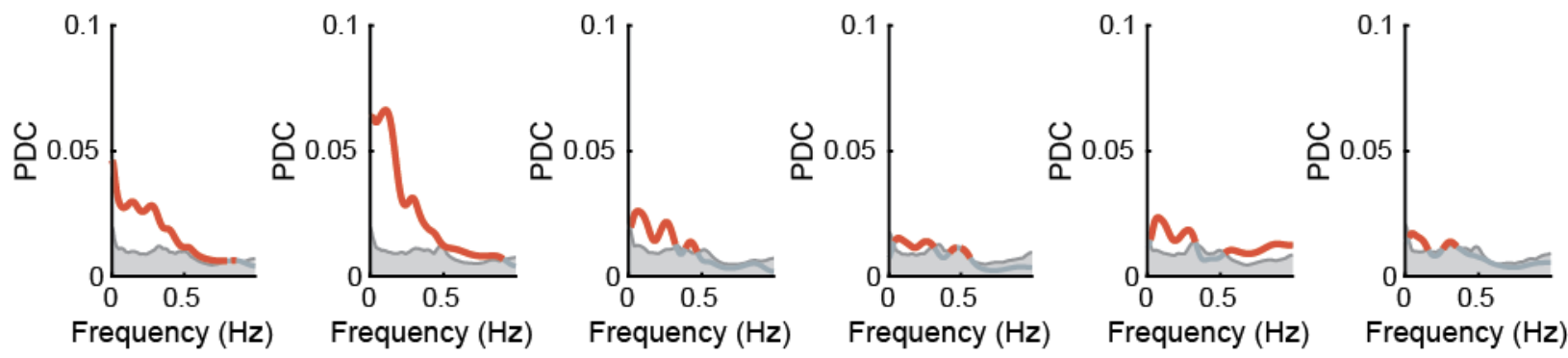

Blink to brain

Body to brain

Mouth to brain

Ear to brain

Arm to brain

Foot to brain
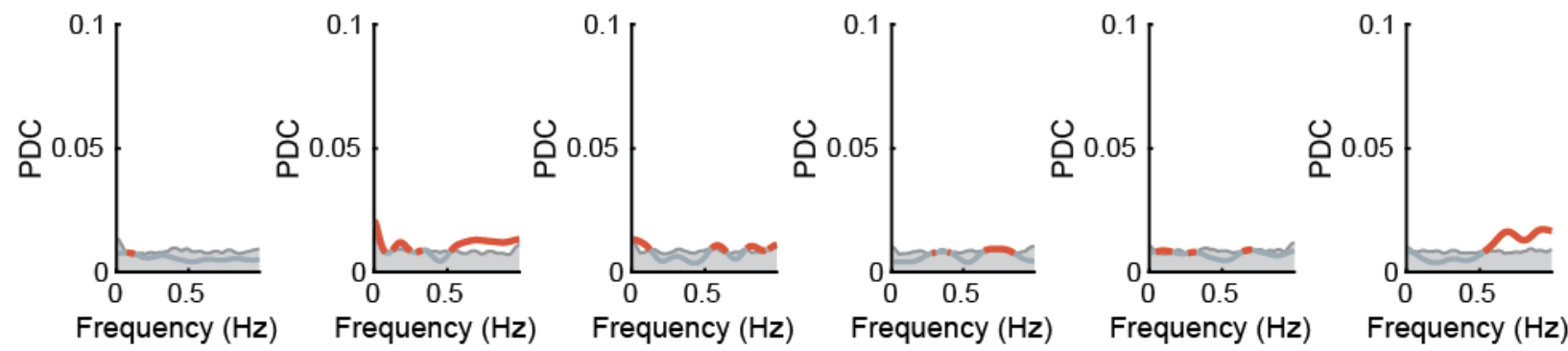

Fig. S4. bPDC between ROIs and each type of movement. The bPDC was among three subsets: ROIs, types of movements, HR. Red highlights values significantly higher than the $95 \% \mathrm{CI}$ of phase-randomized surrogates. 
A

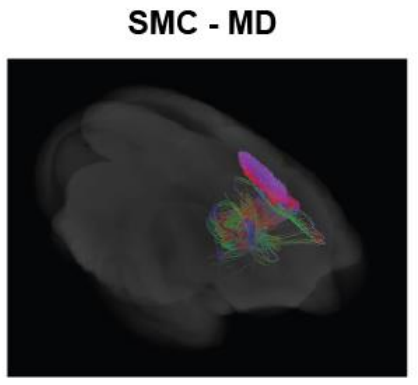

vmPFC - LSD

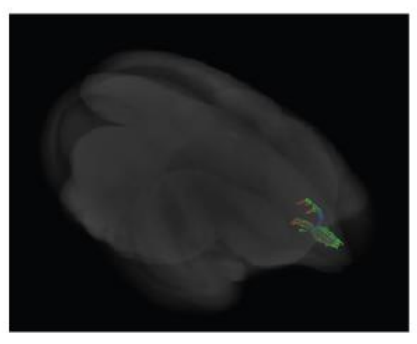

B

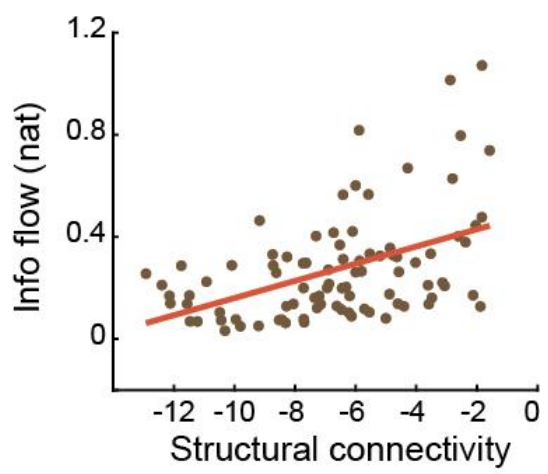

1

2

3

4

5

6

Fig. S5. Functional connectivity correlates with structural connectivity. (A) Examples of fiber tractography. (B) Information flow (symmetrized) vs. log-probabilistic tractography.

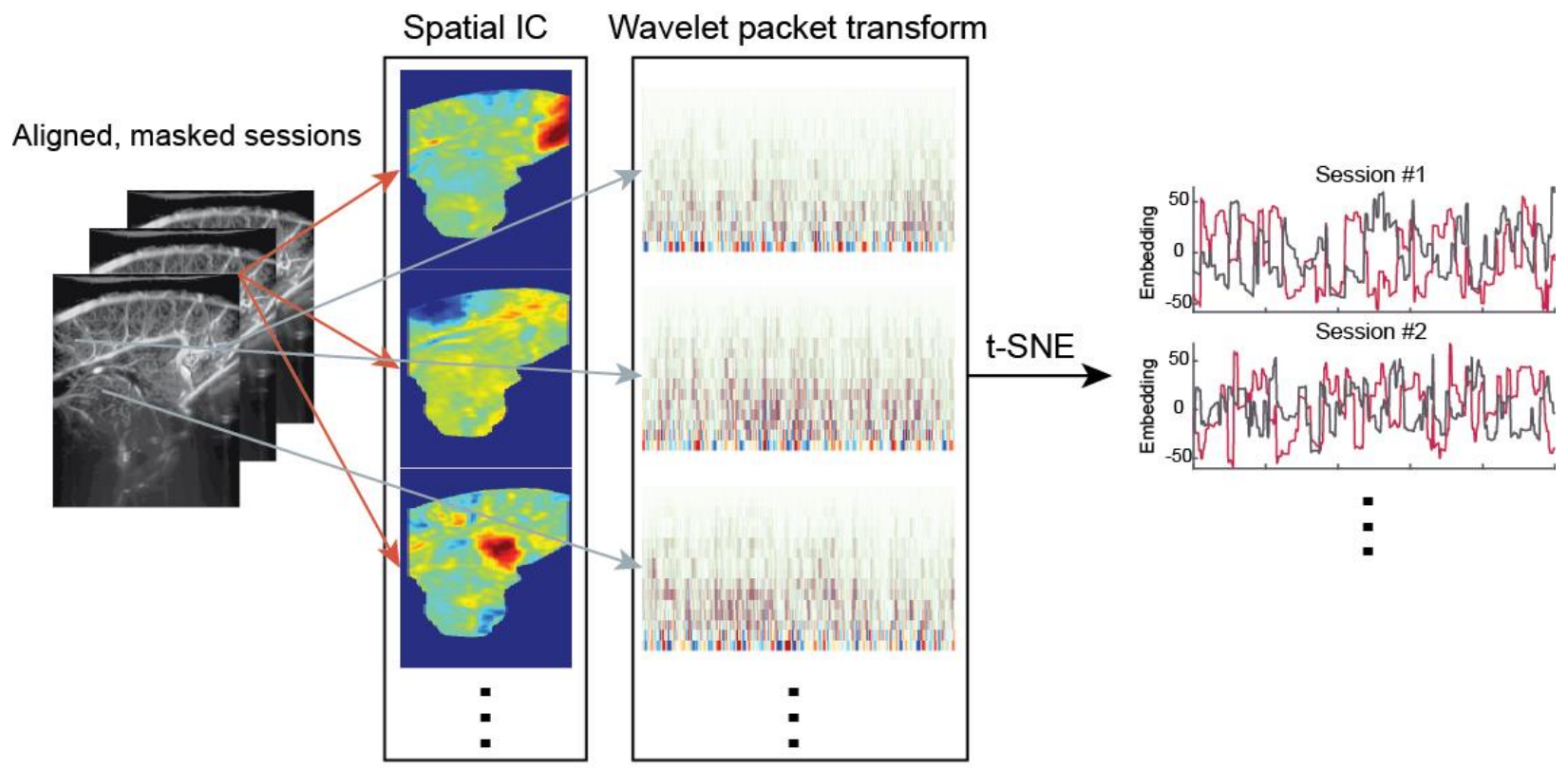

Fig. S6. Dimensionality reduction process. 

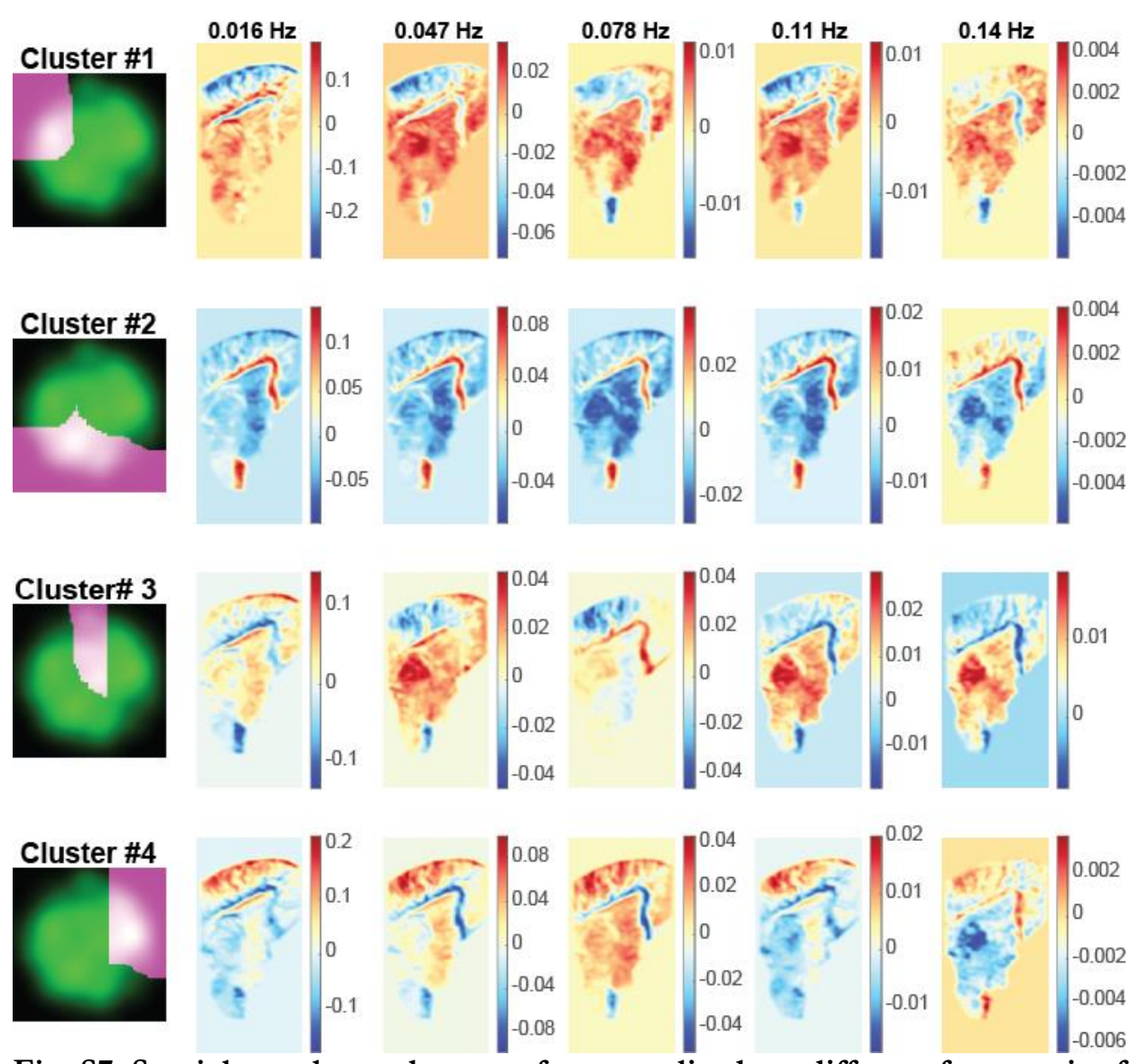

Fig. S7. Spatial wavelet packet transform amplitude at different frequencies for each cluster.
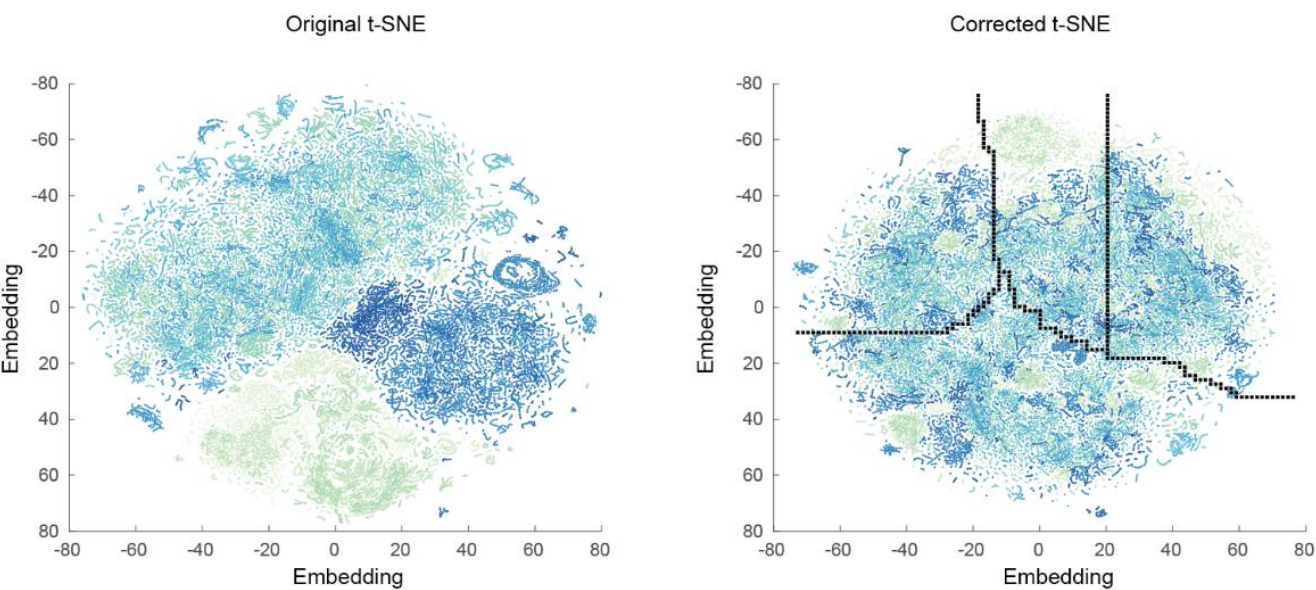

Fig. S8. t-SNE before and after correction for session dependence. Color represents session number.

\section{Proof of PDC's invariance to causal filtering}

Given the Fourier transform of a multivariate time-series $X(\omega)$, applying any causal filtering $G(\omega)$ gives filtered signals $Y(\omega)=G(\omega) X(\omega)$. In the frequency domain, $X(\omega)$ can be written in a moving average representation $X(\omega)=H(\omega) w(\omega)$, where $H(\omega)$ stands for the moving average coefficient and $w(\omega)$ is the Fourier transform of the zero-mean innovation process. Hence we have $Y(\omega)=G(\omega) H(\omega) w(\omega)$. Assuming $H$ and $G$ are invertible, we can further write $H^{-1}(\omega) G^{-1}(\omega) Y(\omega)=w(\omega)$. 
By definition, the PDC from $j \rightarrow i$ is $\pi_{i j}(\omega) \equiv \frac{\left|\hat{A}_{i j}(\omega)\right|}{\sqrt{\sum_{k=1}^{N} \hat{A}_{k j}(\omega) \hat{A}_{k j}^{*}(\omega)}}$, where the matrix $\hat{A}(\omega)=I-$

$2 A(\omega)$ and $A(\omega)$ is the autoregression coefficient and $N$ the total number of signals. It is known that $\hat{A}(\omega)=$

$3 \quad H^{-1}(\omega)$ and hence we have $\hat{A}(\omega) G^{-1}(\omega) Y(\omega)=w(\omega)$. As $G$ is diagonal (otherwise inter-dependence is

4 artificially introduced), element-wise we have $\tilde{A}_{i j}(\omega)=\hat{A}_{i j}(\omega) G_{j}^{-1}(\omega)$. The new PDC of $Y$ is then

$5 \quad \tilde{\pi}_{i j}(\omega)=\frac{\left|\tilde{A}_{i j}(\omega)\right|}{\sqrt{\sum_{k=1}^{N} \tilde{A}_{k j}(\omega) \tilde{A}_{k j}^{*}(\omega)}}=\frac{\left|\hat{A}_{i j}(\omega) G_{j}^{-1}(\omega)\right|}{\sqrt{\sum_{k=1}^{N} \hat{A}_{k j}(\omega) G_{j}^{-1}(\omega) G_{j}^{-1 *}(\omega) \hat{A}_{k j}^{*}(\omega)}}=\pi_{i j}(\omega)$. Q.E.D. 